\title{
Affective picture processing: The late positive potential is modulated by motivational relevance
}

\author{
HARALD T. SCHUPP, ${ }^{\mathrm{a}}$ BRUCE N. CUTHBERT, ${ }^{\mathrm{a}}$ MARGARET M. BRADLEY, \\ JOHN T. CACIOPPO, ${ }^{\mathrm{b}}$ TIFFANY ITO, ${ }^{\mathrm{c}}$ AND PETER J. LANG ${ }^{\mathrm{a}}$ \\ ${ }^{a}$ NIMH, Center for the Study of Emotion and Attention, University of Florida, Gainesville, USA \\ ${ }^{\mathrm{b}}$ Department of Psychology, University of Chicago, Chicago, Illinois, USA \\ ${ }^{\mathrm{c}}$ Department of Psychology, University of Colorado, Boulder, USA
}

\begin{abstract}
Recent studies have shown that the late positive component of the event-related-potential (ERP) is enhanced for emotional pictures, presented in an oddball paradigm, evaluated as distant from an established affective context. In other research, with context-free, random presentation, affectively intense pictures (pleasant and unpleasant) prompted similar enhanced ERP late positivity (compared with the neutral picture response). In an effort to reconcile interpretations of the late positive potential (LPP), ERPs to randomly ordered pictures were assessed, but using the faster presentation rate, brief exposure $(1.5 \mathrm{~s})$, and distinct sequences of six pictures, as in studies using an oddball based on evaluative distance. Again, results showed larger LPPs to pleasant and unpleasant pictures, compared with neutral pictures. Furthermore, affective pictures of high arousal elicited larger LPPs than less affectively intense pictures. The data support the view that late positivity to affective pictures is modulated both by their intrinsic motivational significance and the evaluative context of picture presentation.
\end{abstract}

Descriptors: Emotion, ERP, Affective evaluation, Late positive potential, Slow wave, Motivated attention

Research by Cacioppo and associates (Cacioppo \& Berntson, 1994; Cacioppo, Crites, Berntson, \& Coles, 1993; Cacioppo, Crites, \& Gardner, 1996; Cacioppo, Crites, Gardner, \& Berntson, 1994) has shown that scalp-recorded, event-related potentials (ERPs) vary with the judged emotionality of picture stimuli. Specifically, a widely distributed, late positive potential (LPP) is enhanced for stimuli evaluated as distant from an established affective context. Thus, a pleasant target stimulus presented within a series of unpleasant pictures elicits a larger LPP than does the same pleasant target, presented among other pleasant stimuli. Similar results are found for unpleasant targets (in a pleasant series) for this affective oddball paradigm (Cacioppo et al., 1993; Gardner, Cacioppo, Berntson, \& Crites, in press). Furthermore, the greater the affective

Some of these data were presented in a poster at the 37th convention of the Society for Psychophysiological Research in Cape Cod, Massachusetts, USA (Schupp, Cuthbert, Bradley, McManis, Hillman, Cacioppo, \& Lang, 1997).

This work was supported in part by National Institute of Mental Health grants MH37757, MH43975, and MH52384 to P.J.L.; and by a postdoctoral grant from the Deutsche Forschungsgemeinschaft (Schu 1074/2-1) to H.S.

Harald Schupp is now at the University of Greifswald, Germany; Bruce Cuthbert is now at the National Institute of Mental Health; and Tiffany Ito is now at the University of Colorado.

We thank Charles Hillman and Mark McManis for their assistance.

Address reprint requests to: Peter J. Lang, NIMH Center for the Study of Emotion and Attention, Box 100165 HSC, Gainesville, FL 32610-0165, USA. E-mail: langlab@nersp.nerdc.ufl.edu; or Harald Schupp, University of Greifswald, Department of General and Physiological Psychology, FranzMehring-Str. 47, 17489 Greifswald, Germany. distance of a target (the greater its valence difference from the series) the larger the late potential (e.g., Cacioppo et al., 1994). These findings appear to parallel results obtained with conventional, nonaffective oddball tasks, in which a rare stimulus event (e.g., a high tone preceded by a series of low tones) elicits larger late positivity (P300) than a stimulus consistent with the context (Donchin \& Coles, 1988). The LPP in the affective oddball paradigm differs somewhat from the traditional, nonaffective P300 in that it usually occurs later, and appears to be partially lateralizedwith larger LPP amplitudes over the right than the left parietal hemisphere (Cacioppo et al., 1996).

A different picture perception paradigm has been used by several other investigators in which pleasant, neutral, and unpleasant pictures appear with equal probability in a random sequence (e.g., Cuthbert et al., 1995; Cuthbert, Schupp, Bradley, Birbaumer, \& Lang, in press; Diedrich, Naumann, Maier, Becker, \& Bartussek, 1997; Palomba, Angrilli, \& Mini, 1997; Schupp et al., 1996). Unlike the oddball studies, pictures are usually presented for a sustained period, and often with an intertrial interval (ITI) of many seconds. In this procedure, substantial late positive shifts in the ERPs are evoked by emotional pictures (both pleasant and unpleasant), compared with neutral images, similarly over left and right lateral recording sites. This positive shift can begin as early as $200 \mathrm{~ms}$ after onset for pleasant pictures (somewhat later for unpleasant pictures) and may be sustained over a long period of picture presentation. The phenomenon is accentuated for pleasant and unpleasant pictures of high emotional intensity/arousal (e.g., Cuthbert et al., in press), consistent with the view that emotional pictures engage basic motivational circuits in the brain (Lang, Bradley, \& Cuthbert, 1997). 
These two paradigms share a common result: Enhanced late positivity for selected, affective stimuli. In the previous studies by Cacioppo and colleagues, however, the observed differences between affective pictures were seen to depend importantly on the oddball status of the target affective stimulus. In research using random picture presentation, the larger late positivity to emotional stimuli, compared with neutral stimuli, has appeared to be an intrinsic reflection of emotional processing. The paradigms also differ in their temporal parameters: In the oddball studies, pictures are exposed briefly and at a relatively rapid rate; in contrast, with random presentation, pictures are often shown for a longer periods (e.g., $6 \mathrm{~s}$ ) and with longer ITIs.

The present experiment was designed to determine if brain potentials are modulated by the intrinsic emotional properties of pictures when they are presented briefly and in fixed, rapid sequences, but without an oddball manipulation. Thus, pleasant, neutral, and unpleasant pictures were presented in a random order, as in studies examining implicit ERP responses to affective stimuli (e.g., Cuthbert et al., 1998). However, the rate and duration of stimulus presentation paralleled the modified oddball task used in attitude research (Cacioppo et al., 1993), that is, stimuli were shown for $1.5 \mathrm{~s}$ in distinct sequences of six pictures. It was predicted that the late components of the ERP would still show larger positive potentials for both pleasant and unpleasant pictures, compared with neutral picture stimuli, and that this effect would increase with a picture's greater affective intensity/arousal. Finally, the hypothesis was tested that LPPs for emotionally arousing pictures are more pronounced at right parietal electrodes than at the left hemisphere site-analogous to Cacioppo et al.'s (1996) laterality findings for the LPP and affective distance.

\section{Method}

\section{Subjects}

Twenty-eight volunteers (16 female) from the University of Florida introductory psychology course participated as part of a class requirement. The data from five subjects were excluded from analysis because of computer failure $(n=3)$ or excessive EEG artifacts $(n=2)$, leaving 23 participants for the final sample.

\section{Stimuli and Procedure}

Sixty color pictures were chosen from the International Affective Picture System (IAPS) (Lang, Bradley, \& Cuthbert, 1999), depicting 20 unpleasant events (e.g., snakes, mutilations, etc.), 20 pleasant events (e.g., sports, opposite sex nudes, etc.), and 20 neutral events (e.g., neutral faces, household objects, etc.). ${ }^{1}$ The three categories differed significantly from each other in IAPS normative valence ratings. Arousal ratings did not differ for pleasant and unpleasant contents, but mean arousal levels for both emotional categories were significantly higher than for neutral contents. Half of the subjects viewed a slightly different slide set. Ten of the unpleasant pictures were replaced with unpleasant words and symbolic signs. The data for these symbolic stimuli were excluded from all analyses, resulting in 150 rather than 180 analyzable trials for these subjects. Statistical analysis revealed that the LPP to the pleasant, neutral and unpleasant pictures was not changed by the occasional presentation of symbolic signs in one group.

${ }^{1}$ The slide set contained most of the 54 pictures listed in a previous report (Schupp et al., 1997) except three neutral pictures (7020, 7160, 7180) which were replaced with pictures 7002,7025 , and 7034. Two additional neutral, pleasant, and unpleasant pictures were added: 3110, 3053; 4608, 4653; and 7035, 7190.
Subjects were seated in a recliner in a sound-attenuated room. After attachment of electrodes, task instructions were given. Pictures were displayed for $1.5 \mathrm{~s}$ and presented serially in sequences of six pictures. To ensure that the subject was attending to the pictures, an evaluation task was introduced. After picture offset, the subject was asked to evaluate each picture as either pleasant, neutral, or unpleasant using a three-way response button. ${ }^{2}$ The ITI (including overt response) varied between 1.5 and $3 \mathrm{~s}$. Each sequence of six pictures was initiated by a button press from the subject and a total of 30 sequences were presented. Thus, each picture was viewed three times in the total of 180 trials. The order of pleasant, neutral, and unpleasant pictures was perceptually random within a sequence. According to normative group ratings (Lang et al., 1999), sequences of no more than two pictures of the same valence were allowed. All 60 pictures were presented before repetition of the picture set (in a different order). Each subject received his or her own order of picture presentation. One sequence of six pictures served as practice trials.

\section{Data Collection, Reduction, and Analysis}

The electroencephalogram (EEG) and the vertical and horizontal electrooculogram (EOG) were amplified and bandpass filtered from 0.08 to $35 \mathrm{~Hz}$. Data were collected from $0.5 \mathrm{~s}$ before until $1.5 \mathrm{~s}$ after picture onset with a sampling rate of $250 \mathrm{~Hz}$. EEG was recorded from $\mathrm{F} 3, \mathrm{Fz}, \mathrm{F} 4, \mathrm{C} 3, \mathrm{C} 4, \mathrm{P} 3, \mathrm{Pz}, \mathrm{P} 4, \mathrm{~A} 1$, and $\mathrm{A} 2$, all referenced to $\mathrm{Cz}$, and converted offline to a linked mastoid reference. All individual trials with EEG or EOG activity above \pm $100 \mu \mathrm{V}$ were excluded from further analysis, and the EEG was then corrected for vertical and horizontal eye movement artifacts (Miller, Gratton, \& Yee, 1988). Only EEG artifact-free trials with congruent subjective and normative categorization of the pictures were accepted. ${ }^{3}$

Preliminary analysis investigated the LPP peak and a LPP area measure in a window lasting from 350 to $750 \mathrm{~ms}$ after stimulus onset. Both analyses converged with regard to the affective modulation of the ERPs. However, LPP peaks were difficult to score at some leads, and individual subjects. Thus, results of the LPP area measure are reported here. A multivariate analysis of variance containing the factors affect (pleasant, neutral, unpleasant), caudality (frontal, central, parietal), and laterality (left, mid, right recording sites) was conducted. For effects involving repeated measures, the Wilks Lambda procedure was used to correct for violations of sphericity.

\section{Results}

\section{$L P P$}

As illustrated in Figure 1 for midline sites, pleasant and unpleasant pictures elicited a generally more pronounced overall positive shift (LPP) compared with neutral pictures, ${ }^{4} F(1,22)=78.1$ and 48.1,

\footnotetext{
${ }^{2}$ Cacioppo and colleagues have used a slightly different evaluative task, suited to the "oddball" paradigm, that is, participants are instructed to count the number of pictures in a series judged to be "pleasant" or "unpleasant."

${ }^{3}$ Five percent of trials were rejected due to artifact, and $25 \%$ due to miscategorization, largely due to a few subjects who tended to report a priori emotional pictures as neutral. Control analyses including the miscategorized trials showed no differences from the results as reported.

${ }^{4}$ As in earlier studies (Cuthbert et al., 1995), waveforms to pleasant pictures separated significantly earlier from neutral pictures than unpleasant pictures. For brevity, only the LPP modulation is reported here.
} 

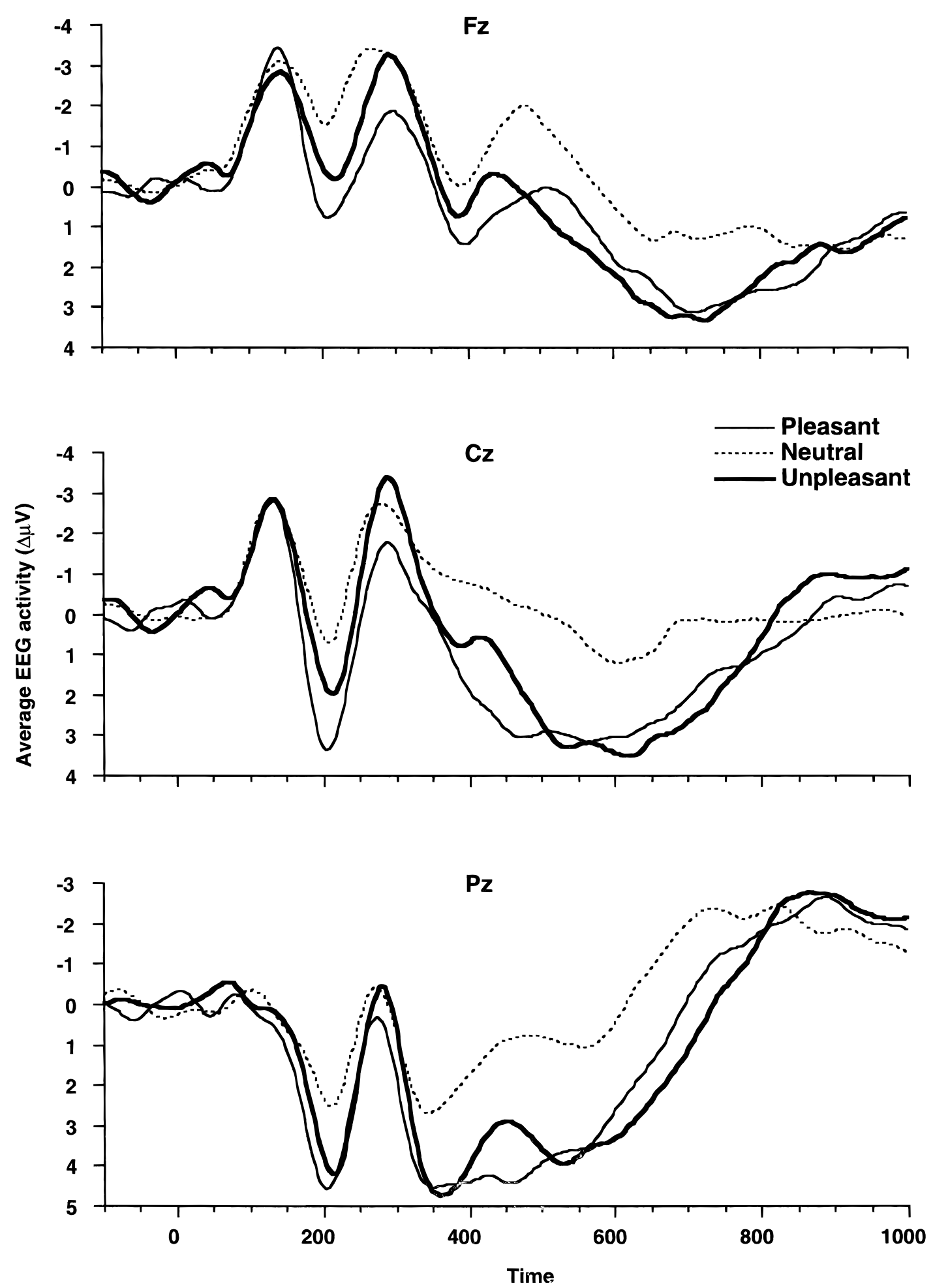

Figure 1. Picture onset synchronized grand-average event-related potential (ERP) waveforms for each valence category (pleasant, neutral, and unpleasant) from midline electrodes $\mathrm{Fz}, \mathrm{Cz}$, and $\mathrm{Pz}$.

$p<.001$, Affect $F(2,21)=39.0, p<.001$. Pleasant and unpleasant pictures did not differ from each other, $F(1,22)=0.1$, $n s$.

Affect interacted with caudality, $F(4,19)=6.0, p<.01$, reflecting a small increase in response to emotional pictures (both pleasant and unpleasant) from frontal to posterior sites (linear trend for emotional pictures: $F[1,22]=6.99, p=.015)$. This trend was not found for neutral pictures. Affect also interacted with laterality, $F(4,19)=4.6, p<.01$ : Overall, the LPPs were not statistically different at left, midline, and right cortical electrodes for both neutral and pleasant pictures. For unpleasant pictures, 
however, the LPPs tended to be somewhat smaller at lateral sites than at the midline, $F(2,21)=14.76, p<.001$.

Separate analyses of variance (ANOVAs) were carried out to determine if, despite these modest variations, the LPP was sensitive to picture emotionality at all sites. Affective modulation was highly significant at frontal, central, and parietal electrodes, affect $F_{\mathrm{s}}(2,21)>37, p<.001$. Specifically, pleasant pictures elicited a larger positive shift compared with neutral contents at frontal, central, and parietal sites, $F_{\mathrm{S}}(1,22)>27,74,90, p<.001$, respectively. Larger LPP amplitudes were also found for unpleasant compared to neutral images at all sites, $F \mathrm{~s}(1,22)>22,46,46, p<$ .001 , respectively. Pleasant and unpleasant pictures evoked comparable LPP amplitudes, $F \mathrm{~s}(1,22)<1$, $n s$.

Separate lateral analysis of electrode sites also showed significant emotional modulation, affect $F(2,21)>32, p<.001$. Again, emotional pictures elicited - at left, mid, and right electrodeslarger LPP amplitudes than neutral contents, $F \mathrm{~s}(1,22)=92.3$, $61.0,67.9, p<.001$, for pleasant pictures; $F \mathrm{~s}(1,22)=40.2$, 48.6, $43.8, p<.001$, for unpleasant pictures. There were no laterality differences in LPP amplitude between pleasant and unpleasant pictures. To summarize the above findings, emotional pictures showed more late positivity than neutral pictures at all recorded electrode sites.

A significant overall effect of caudality, $F(2,21)=19.4, p<$ .001, indicated that LPP amplitudes were generally larger at central than frontal leads, $F(1,22)=28.0, p<.001$, and intermediate at parietal sites. Caudality and laterality interacted, $F(4,19)=7.3$, $p<.001$ : At parietal leads only, larger amplitudes were found at the midline $(\mathrm{Pz})$ than at lateral sites $(\mathrm{P} 3$ and $\mathrm{P} 4), F(1,22)>6.6$, $p<.05)$.

\section{Emotional Intensity}

The effect of a modulation of the LPP as a function of emotional intensity was assessed for midline sites. The emotional pictures (pleasant and unpleasant) were sorted by normative arousal ratings into high and low arousal subsets. In analyzing the picture subset differences, only subjects viewing the full picture set were used $(n=10)$. Pleasant and unpleasant pictures of high emotional arousal elicited larger LPPs $(M=2.0 \mu \mathrm{V})$ than less arousing affective pictures $(M=.6 \mu \mathrm{V}), F(1,9)=24.1, p<.001$. Again, LPP did not differ between pleasant and unpleasant slides, $F(1,9)=1.9, n s$. However, the modulation of emotional intensity varied with caudality, $F(2,18)=7.8, p<.05$. Emotional intensity effects were observed at all sites, but were most pronounced at $\mathrm{Cz}$ and $\mathrm{Pz}$, $F_{\mathrm{S}}(1,9)=5.5,27.0$, and 37.0, $p \mathrm{~s}<.05$ for $\mathrm{Fz}, \mathrm{Cz}$, and $\mathrm{Pz}$, respectively.

\section{Discussion}

Consistent with the results from earlier studies with the picture perception paradigm (e.g., Cuthbert et al., 1995; Palomba et al., 1997), emotional pictures (either pleasant or unpleasant) evoked a larger LPP than neutral materials. The positive amplitudes to affective pictures were similar (though less sustained) to those observed previously for a 6-s presentation (Cuthbert et al., in press). The statistical effects were very robust. A simple difference score indicated that all 23 participants showed a larger positive potential at $\mathrm{Pz}$ to pleasant than to neutral pictures $(\Delta=2.6 \mu \mathrm{V})$. A similar effect was observed for unpleasant compared with neutral pictures (22 of 23 participants, $\Delta=2.4 \mu \mathrm{V}$ ). In addition, the LPP was more pronounced for highly arousing material (e.g., erotic or violent content). That is, pleasant and unpleasant pictures of high emotional intensity elicited larger positive waves than less intense pleasant and unpleasant stimuli. Lang et al. (1997) hypothesized that this sustained positivity reflects motivational engagement, and a commitment of attentional resources to the picture viewing task (cf. Cuthbert et al., 1998; Lang et al., 1998).

The fact that pleasant and unpleasant stimuli are both highly salient in natural environments might explain why the LPP modulation is silent with regard to affective direction. Indeed, the late waveform patterns for pleasant and unpleasant pictures were remarkably similar. On the other hand, it has been shown that ERP waveforms for pleasant pictures differ from neutral contents earlier in time (around $200 \mathrm{~ms}$ ) than do unpleasant contents (e.g., Cuthbert et al., in press). These observations suggest that there may be differences in the activation of appetitive and defensive motivational systems (cf. Cacioppo \& Berntson, 1994) than can be detected by scalp electrodes.

The present study shares many features of the affective oddball task, with respect to stimulus material, stimulus rate, and stimulus duration. One difference concerns the random presentation of pleasant, neutral, and unpleasant stimuli. A nonrandom stimulus presentation serves in the affective oddball task to induce a hedonic context. Affectively inconsistent stimuli elicit substantially larger LPPs, which is conceptualized as due to a mismatch between the hedonic context and affective evaluation of the foreground stimuli (e.g., Cacioppo et al., 1993; Crites, Cacioppo, Gardner, \& Berntson, 1995). This index of affective distance depends on the presence of a hedonic context, that is, repeated presentation of pleasant or unpleasant stimuli (cf. Bradley, Cuthbert, \& Lang, 1996). Thus, LPPs appear to be sensitive to both the intrinsic affective properties of the picture, and the local affective context in which the picture is presented.

The oddball paradigm also appears to be relevant for the finding of differential LPP amplitudes over lateral leads. In affective distance studies, a larger overall amplitude over the right than the left hemisphere was found when subjects were instructed to categorize stimuli for affect (i.e., count the number of "pleasant" or "unpleasant" stimuli in a series). In contrast, it was not found in the absence of this categorization task. In the present research, which included affective categorization but not as a counting task in an oddball paradigm, no hemispheric differences were found at parietal leads. These data suggest that lateralization may depend on the type of emotional categorization and may also depend on stimulus presentation rate or other aspects of task demand.

In summary, the present study shows that pleasant and unpleasant pictures recruit selective attention processes that are reflected in sustained late positive ERP potentials. This effect appears to depend here on the motivational significance of these stimuli (Lang et al., 1997): It is not found for neutral pictures, and furthermore, within affective categories, positive LPP amplitudes increase with emotional stimulus intensity (i.e., arousal). In our other research (e.g., Cacioppo et al., 1996), a qualitatively different modulation of these responses was obtained by systematically varying the evaluative distance of target pictures and the affective context of picture presentation. Although the present study is not an exhaustive, parametric attempt to compare these two paradigms, the data indicate clearly that evocation of differential LPPs is not dependent on stimulus duration, content grouping, or stimulus presentation rate. The collective findings suggest that the intrinsic motivational relevance of affective pictures, as well as the contextual paradigm, can modulate late ERPs to affective stimuli. 


\section{REFERENCES}

Bradley, M. M., Cuthbert, B. N., \& Lang, P. J. (1996). Picture media and emotion: Effects of a sustained affective context. Psychophysiology, $33,662-670$.

Cacioppo, J. T., \& Berntson, G. G. (1994). Relationships between attitudes and evaluative space: A critical review, with emphasis on the separability of positive and negative substrates. Psychological Bulletin, 115, 401-423.

Cacioppo, J. T., Crites, S. L., Jr., Berntson, G. G., \& Coles, M. G. H (1993). If attitudes affect how stimuli are processed, should they not affect the event-related brain potential? Psychological Science, 4, $108-112$.

Cacioppo, J. T., Crites, S. L., \& Gardner, W. L. (1996). Attitudes to the right: Evaluative processing is associated with lateralized late positive event-related brain potentials. Personality and Social Psychology Bulletin, 22, 1205-1219.

Cacioppo, J. T., Crites, S. L., Jr., Gardner, W. L., \& Berntson, G. G. (1994). Bioelectrical echoes from evaluative categorization: I. A late positive brain potential that varies as a function of trait negativity and extremity. Journal of Personality and Social Psychology, 67, 115-125.

Crites, S. L., Jr., Cacioppo, J. T., Gardner, W. L., \& Berntson, G. G. (1995). Bioelectrical echoes from evaluative categorization: II. A late positive brain potential that varies as a function of attitude registration rather than attitude report. Journal of Personality and Social Psychology, 68, 997-1013.

Cuthbert, B. N., Schupp, H., McManis, M., Hillman, C., Bradley, M. M., \& Lang, P. J. (1995). Cortical slow waves: Emotional perception and processing. Psychophysiology, 32, S26.

Cuthbert, B. N., Schupp, H. T., Bradley, M. M., Birbaumer, N., \& Lang, P. J. (in press). Brain potentials in affective picture processing: Covariation with autonomic arousal and affective report. Biological Psychology.

Diedrich, O., Naumann, E., Maier, S., Becker G., \& Bartussek, D. (1997). A frontal positive slow wave in the ERP associated with emotional slides. Journal of Psychophysiology, 11, 71-84.
Donchin, E., \& Coles, M. G. (1988). Is the P300 component a manifestation of context updating? Behavioral and Brain Sciences, 11, 357-427. Gardner, W. L., Cacioppo, J. T., Berntson, G. G. , \& Crites, S. L., Jr. (in press). Distinguishing hostile from hospitable events: Individual differences in evaluative categorizations as indexed by a late positive brain potential. Social Cognition.

Lang, P., Bradley, M. M., \& Cuthbert, B. N. (1997). Motivated attention: Affect, activation, and action. In P. Lang, R. F. Simons, \& M. Balaban (Eds.), Attention and orienting: Sensory and motivational processes (pp. 97-136). Hillsdale, NJ: Erlbaum.

Lang, P. J., Bradley, M. M., \& Cuthbert, B. N. (1999). International Affective Picture System: Instruction manual and affective ratings. Technical Report A-4, The Center for Research in Psychophysiology, University of Florida.

Lang, P. J., Bradley, M. M., Fitzsimmons, J. R., Cuthbert, B. N., Scott, J. D., Moulder, B., \& Nangia, V. (1998). Emotional arousal and activation of the visual cortex: An fMRI analysis. Psychophysiology, 35, 199-210.

Miller, G. A., Gratton, G., \& Yee, C. M. (1988). Generalized implementation of an eye movement correction procedure. Psychophysiology, 25, 241-243.

Palomba, D., Angrilli, A., \& Mini, A. (1997). Visual evoked potentials, heart rate responses and memory to emotional pictorial stimuli. International Journal of Psychophysiology, 27, 55-67.

Schupp, H. T., Cuthbert, B. N., Bradley, M. M., McManis, M. M., Hillman, C., Cacioppo, J., \& Lang, P. J. (1997). Quick pics: Emotional pictures and cortical ERPs [Abstract]. Psychophysiology, 34, S80.

Schupp, H. T., Cuthbert, B. N., Hillman, C., Raymann, R., Bradley, M. M., \& Lang, P. J. (1996). ERPs and blinks: Sex differences in response to erotic and violent picture content [Abstract]. Psychophysiology, 33, S75. 